\title{
Analysis of the arginine biosynthetic gene cluster argCJBDFR of Corynebacterium crenatum
}

\author{
Haitao Jiao ${ }^{1}$, Yong Yuan ${ }^{2}$, Yonghua Xiong ${ }^{2}$, Xuelan Chen ${ }^{1}$ \\ ${ }^{1}$ College of life sciences, Jiangxi Normal University, Nanchang, China; \\ ${ }^{2}$ State Key Laboratory of Food Science and Technology, Nanchang University, Nanchang, China. \\ Email: yhxiongchen@163.com, xuelanchen14@yahoo.com.cn
}

Received 8 October 2010; revised 28 October 2010; accepted 8 November 2010.

\begin{abstract}
Objective: Corynebacterium crenatum AS1.542, a Gram-positive bacterium and indigenous nonpathogenic corynebacteria, is widely exploited for the industrial production of amino acids. The objective of this paper is to clarify the genetic information of the arginine biosynthetic pathway, and further more contribute to the improvement of arginine production. Methods: Polymerase chain reaction (PCR) technology was employed for obtaining the arginine biosynthetic gene sequence and softwares eg. Lasergene, BPROM, RNAshapes were used for the analysis of obtained sequences. Results: Arginine biosynethetic gene cluster of $C$. crenatum, comprising $\arg J, \arg B, \arg D, \arg F, \arg R$ and part of $\arg C$, has been amplified and sequenced. The gene order has been established as $\operatorname{argCJBDFR}$, with a entire length of $6.08 \mathrm{~kb}$. Conclusion: An internal promoter was found in the upstream of $\arg B$ gene, four $\operatorname{argBDFR}$ ORFs are located in a same transcription unit, and the transcripiton termination of $\arg C$ gene is irrelevant with the rho-factor. Comparison with ornithine acetyltransferase (coded by argJ gene) from $C$. glutamate, ornithine acetyltransferase from $C$. crenatum also belongs to the monofunctional enzymes.
\end{abstract}

Keywords: Corynebacterium crenatum; $\operatorname{argCJBDFR}$ Sequence; Ornithine Acetyltransferase; $\arg R$ Gene

\section{INTRODUCTION}

Arginine biosynthesis commences with the acetylation of the amino group of glutamate (Figure 1). Eight enzymes coded by eight or nine genes take part in the process of catalyzation, resulting the biotransformation of glutamate into arginine [1]. The pathway of arginine biosynthesis can be divided into two parts according to two strategies evolved in the removal of the acetyl group. One is called the "linear" pathway, in which $\operatorname{argE}$ gene coded acetylornithinase catalyses the hydrolysis of $\mathrm{N}$-acetylornithine into the arginine precursor ornithine and acetate; the other is called the "economic cyclic" pathway, in which acetylornithine is catalyzed into ornithine and acetyl groups, and recycled with generation of acetylglutamate by $\arg J$ gene coded ornithine acetyltransferase [2]. ArgJ has both acetylornithinase (coded by $\arg E$ ) function and $\mathrm{N}$-acetylglutamate synthase (coded by $\operatorname{argA}$ ) functions in the "cyclic" pathway. Literatures showed that Enterobacteriaceae and Sulfolobus solfataricus [2,3] adopted the "linear" pathway in which the metabolic flow is controlled by arginine-mediated feedback inhibition of the first biosynthetic step; all the other prokaryotes, including Methanogenic archaea, Neisseria gonorrboese, members of the genus Bacillus and the eukaryotic microbes, use the "cyclic" pathway in which the metabolic flow was controlled by arginine-mediated feedback inhibition of the second biosynthetic step or by orthinine-mediated feedback inhibition of the fifth step [2]. Although the argJ gene by itself would thus be able to assure both the first and the fifth steps of arginine biosynthesis in above mentioned organisms, there is genetic evidence for the existence of the cloned ornithine acetyltransferase genes from Pseudomonas aeruginosa [4], Saccbaromyces cerevisiae [5], Streptomyces coelicolor [6] and Corynebacterium glutamicum complementing E. coli $\operatorname{argE}$ but not $\operatorname{argA}$ mutants.

C. crenatum AS1.542, a Gram-positive bacterium and indigenous nonpathogenic corynebacteria, is widely exploited for the industrial production of amino acids. The genetic information of arginine biosynthetic pathway was analyzed and clarified in this paper with the aim to contribute to the improvement of arginine production.

\section{MATERIALS AND METHODS}

\subsection{Reagent}

All the primers were synthesized by Shanghai Biotech- 


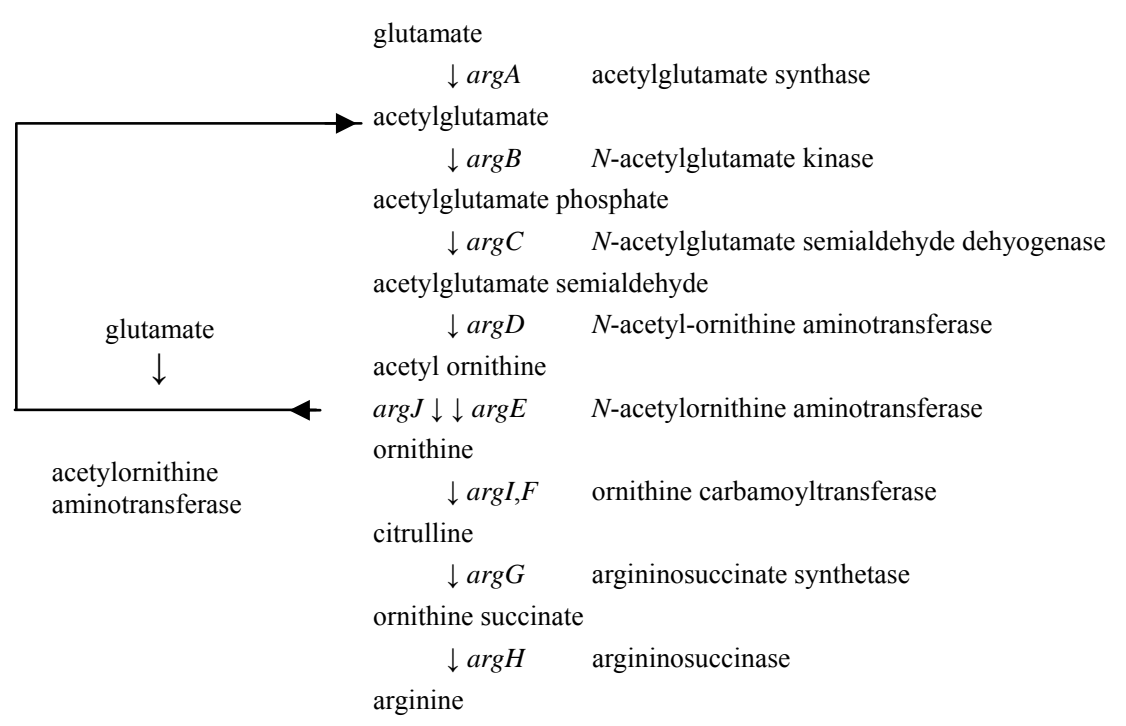

Figure 1. The "linear" (in E. coli) and the "alternative cyclic" (in B. stearothermophilus and C. glutamate) arginine biosynthesis pathways.

nology Corporation, China. Gel extraction kit and pGEMT-Easy vector were purchased from Promega, USA.

\subsection{Bacterial Cultivation}

C. crenatum and C. glutamicum were cultured in a rotary shaker incubator at $150 \mathrm{rpm}$ under $30^{\circ} \mathrm{C}$ in Luria-Bertani (LB) medium.

\subsection{DNA Manipulation, Amplification, Sequencing and Analysis}

Chromosomal DNA of C. crenatum was isolated as described by Shengdong L [7]. Total genomic DNA (50 ng) was used as a template for PCR amplification of $\operatorname{argCJBDFR}$ gene cluster. The employed primers were designed using the conserved sequences of C. glutamicum ATCC 13032, C. diphtheriae gravis NCTC13129, C. efficiens YS-314 and Mycobacterium tuberculosis CDC 1551. The primers were: sense-1 (5'-TCAAGGTTGCA ATCGCAGGAGCC-3'), antisense-1 (5'-GCAACTCAC CAATAAGACCAGTGG-3'), sense-2 (5'-CCGCAGCG CCGTGTTTACACGTAACC-3'), antisense-2 (5'-GAC AAGATTGTTGTCGTGAAATATG-3'), sense-3 (5'-AT CTTTGGAATCATGCCGGAATC-3'), antisense-3 (5'TCTTCGTCGGTGATCACCAGCGG-3'), sense-4 (5'CATGCCAGATTCTGGCTGATCTGCAG-3') and antisense-4 (5'-GCAAGAACGATGCGGTTAGTCATG-3'), respectively. The amplicons were purified using gel extraction kit and sub-cloned into pGEM-T-Easy vector. The selected clones were subjected to sequencing of $\operatorname{argCJBDFR}$ gene cluster fragments with SP6 and T7 sequencing primers using $\mathrm{ABI}$ prism 3730 sequencer.

The sequence data were compiled, aligned and analyzed using Lasergene software (DNASTAR), Soft- berry's BPROM (www.softberry.com) and RNAshapes WebServices (BiBiServ) et al.

\section{RESULTS AND DISCUSSION}

\subsection{PCR Amplification}

The results of PCR amplification were shown in Figure 2. The full-length of amplified DNA, aligned with SeqMan function of Lasergene software, was $6080 \mathrm{bp}$. The full-length DNA sequence alignment of $C$. crenatum

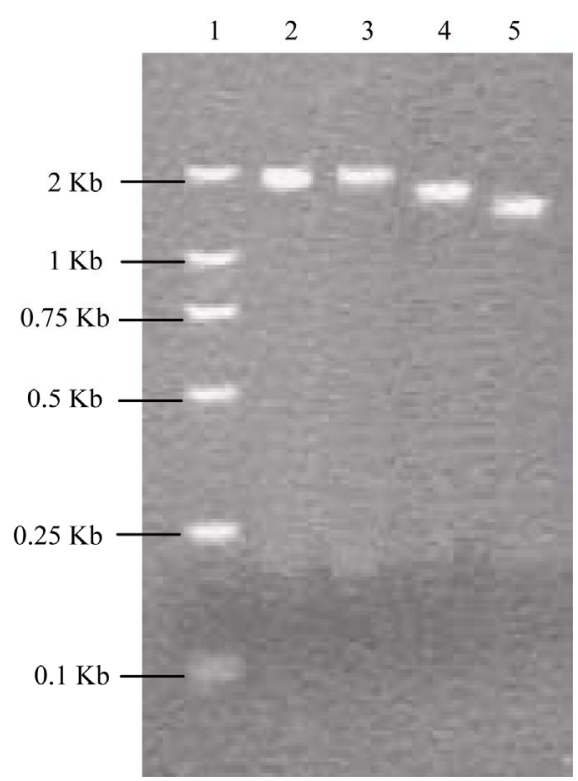

Figure 2. PCR amplification of tandem arginine biosynthetic genes (Lane 1: DL2000 Marker; lane 2, 34 and 5: $\arg C J, \operatorname{argJBD}$, $\operatorname{argDF}$ and $\operatorname{argFR}$, respectively). 
showed a very high homology with the arginine biosynthetic gene cluster: $\operatorname{argCJBDFR}$ of C. glutamicum ATCC 13032 by blastn analysis in NCBI. This result indicated that the $6080 \mathrm{bp}$ sequence of $C$. crenatum was the arginine biosynthetic gene cluster. The acession number for the sequence in Genbank is AY509864.

\subsection{Sequence Analysis}

Analysis of the nucleotide sequence revealed the presence of five intact open reading frames (ORFs): $\arg J$, $\arg B, \arg D, \arg F, \operatorname{argR}$, and partial of $\arg C$ ORF (shown in Figure 3).

Sequence analysis of the gene cluster indicated that the $\arg B$ TAA termination coden was contiguous to the initiation codon of the $\arg D$ gene, the distance between $\arg D$ and $\arg F$ was $13 \mathrm{bp}$, and $\arg F$ gene was blocked off $\operatorname{argR}$ by $3 \mathrm{bp}$. This phenomenon suggested that the four ORFs located in the same transcription unit. Two relatively long intergenic spacers were found in the $\operatorname{argC/\operatorname {arg}J}$ and $\operatorname{argJ} / \arg B$, and a potential promoter region existing in the upstream of $\arg B$ gene was doped out, but there was no potential promoter existing in the upstream of $\arg J$ gene. The $\arg B$ upstream sequence was shown in Figure 4. The interval between Sextama and Pribnow shown in under-double-line is $15 \mathrm{bp}$. A pair of inverse repeat sequence indicated by under-single-line is presumed to be operator region, also known as Arg box recognized by control protein.

The Arg box consensus were described as TNTGA ATWWWWATTCANW in E. coli [8], CATGAATAAAA ATKCAAK in B. subtilis [9] and AWTGCATRWWYAT GCAWT in Streptomycetes [10] (where $\mathrm{W}=\mathrm{A}$ or $\mathrm{T}, \mathrm{K}=$ $\mathrm{G}$ or $\mathrm{T}, \mathrm{R}=\mathrm{A}$ or $\mathrm{G}, \mathrm{Y}=\mathrm{T}$ or $\mathrm{C}, \mathrm{N}=$ any base). In Addition, Binding of ArgR homologs to the sites similar to ARG boxes has been reported in Salmonella typhimurium [13] and other Bacillus species (B. licheniformis
[11] and B. stearothermophilus [12]). The most popular base of Arg boxes from the strains mentioned above were $\mathrm{A}$ and $\mathrm{T}$, whereas $\mathrm{G}$ and $\mathrm{C}$ in C. crenatum (as shown in Figure 4). The difference might be related with the control of arginine biosynthesis of corynebacterium and the distance of cognation.

A stem-loop structure was found in the downstream of $\arg C$ gene using RNAshapes tool. Two primary characters of rho-independent terminator were appeared: a reverse repeat sequence and the reverse repeat sequence mostly composed with $\mathrm{G}$ and $\mathrm{C}$. The $\arg C$ terminator region was shown in Figure 5. The downstream of rest genes have no fixed features, which implied that the transcription termination of the rest genes were relevant with the rho-factor.

The C. crenatum argJ sequence, compared with blastp software in NCBI, shares 98.5, 70, 79 and 53\% identical amino acids with the ornithine acetyltransferases (OATase) of C. glutamicum [2], C. diphtheriae gravis [14], C. efficiens [15] and M. tuberculosis [16], respectively. Sakanyan et al. reported that the OATase of $C$. glutamicum had only acetylorthine amidohydrolase function but no transacetylation by cloning of argJ gene for heterologous complementation of $\arg A$ deficiency in E. coli [2]. The CLUSTAL alignment indicated that the similarity covered the whole argJ gene sequence between $C$. crenatum and C. glutamicum. The result indicated that $C$. crenatum argJ coded OATase belongs to monofunctional enzymes.

The C. glutamicum ArgJ molecular mass is $39.8 \mathrm{kDa}$, approximately $3 \mathrm{kDa}$ less than the other known bacterial bifuctional OATases. Sakanyan et al held that the missing 11-12 amino acids at the N-terminus was connected with the transacetylation deficiency via CLUSTAL alignment among C. glutamicum, B. sterothermophilus, B. subilis and Neisseria gonorrboeae [2]. In the present

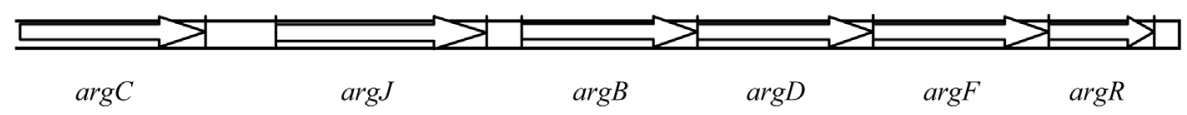

Figure 3. Genetic map of the $6.08 \mathrm{~Kb}$ stretch of C. crenatum A.S1.542 DNA. The long arrowheads indicated the orientation and location of the ORFs.

2090
ATTCCACTGGTGCCCCTGGTGCTCGTGAGGTGGATCTTTCCGGCGCTGACATTGATGTCCG
Pribnow
AATTGATTTGGGCACCAGTGGGGAAGGCCAGGCAACAGTTCGAACCACTGACCTGAGCTT
CTCCTACGTGGAGATCAACTCCGCGTACAGCACTTAAAAAGAAACAACACTCCAACTAACG
AGCAGGGAAAAGGGCACAGCCATG
$\longrightarrow \operatorname{argB}$

Figure 4. Promoter sequence of $\arg B$ gene. 


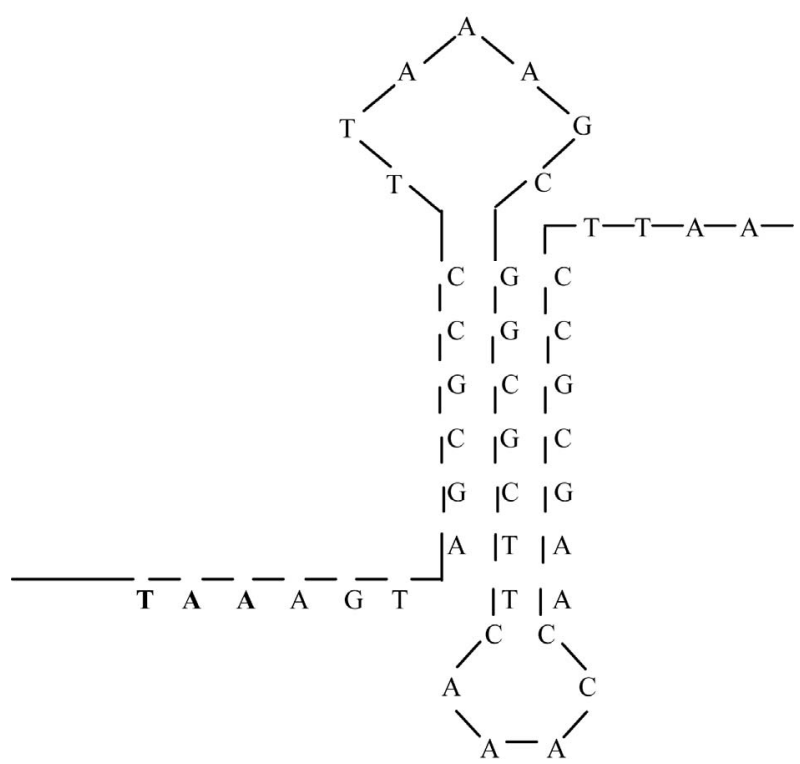

Figure 5. The characters of transcription termination sequence of $\arg C$ gene.

paper, the OATases from 12 strains, containing five OATases (from C. crenatum, C. glutamicum, C. diphteric, C. efficiens and Streptomyces clavuligerus) reported no transacetylation and seven bifunctional OATases, were found that the absence of 11-12 amino acids at the $\mathrm{N}$-terminus had nothing to do with the bifunction (shown in Figure 6).

A Blastp comparison shows that $N$-acetylglutamate kinase (AGKase) polypeptide sequence coded by $\arg B$ gene of $C$. crenatum, 313 amino acids with a predicted molecular mass of $34.6 \mathrm{kDa}$, shares approximately $95 \%$, $63 \%$ and $48 \%$ identical amino acids with those gene of $C$. glutamicum, Bifidobacterium longum [17] and N. meningitides serogroup[18], respectively. AGKase belongs to a member of $\mathrm{Mg}^{2+}$ activated superfamily. The locations of kinases binding-ATP domain are shown in Figure 7.
According to a blastp comparison, $\mathrm{N}$-aetylornithine aminotransferase (AOATase) polypeptide sequence coded by $\arg D$ gene of $C$. crenatum shares approximately 96 , $77.4,61.5$ and $52.2 \%$ identical amino acids with those of C. glutamicum [2], C. efficiens [14], C. diphtheriae [15] and M. tuberculosis [16], respectively. The C. crenatum ArgF sequence shares 97.5, 84.95, 75.5 and $58.1 \%$ identical amino acids with the ornithine carbamoyltransferase from C.glutamicum, C. efficiens, C. diphtheriae and M. tuberculosis, respectively.

The terminal gene in the cluster corresponds to $\arg R$, which is a transcription factor in arginine metabolism. The polypeptide sequence is composed with 172 amino acid residues with a predicted molecular mass of 18.2 $\mathrm{kDa}$. Blastn comparison shows that there are three nucleotide differences between the $\arg R$ of $C$. crenatum and the $\operatorname{argR}$ of C. glutamicum, however, the two ArgR sequences are $100 \%$ identical due to the codon degeneracy. The C. crenatum ArgR sequence shares 71, 89 and $56 \%$ identical amino acids with those of $C$. efficiens, $C$. diphtheriae and M. tuberculosis, respectively.

The ArgR, whose interspace configuration is winged helix-turn-helix (wHTH), consists of a N-terminal DNA-binding domain and a C-terminal oligomerization domain joined by a hinge region $[17,18]$. It was reported that the E. coli $\operatorname{ArgR} \mathrm{C}$-terminal domain contained an arginine pocket defined in part by two aspartic acid residues at positions 128 and $129[13,19]$. By CLUSTAL alignment between the $C$. crenatum $\operatorname{ArgR} \mathrm{C}$-terminus and the E. coli $\operatorname{ArgR} \mathrm{C}$-terminus, it was found that there were two consecutive aspartic acid residues at positions 146 and 147 and eight sequential highly conserved amino acid residues around the two aspartic acid residues in the C. crenatum ArgR C-terminus (shown in Figure 8). Despite there is only $21.2 \%$ identical amino acids between the Gram-positive C. crenatum ArgR and the Gram-negative E. coli ArgR, the important conserved

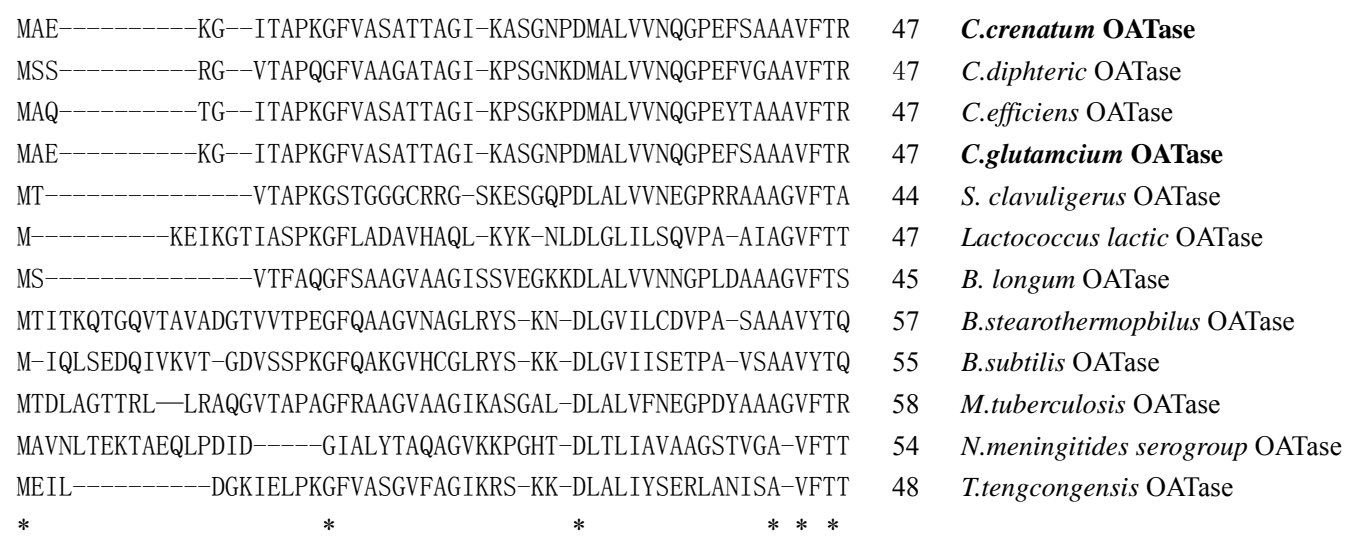

Figure 6. Comparison of N-terminal amino acid sequences of ornithine acetyltransferases by CLUSTAL alignment. (Stars indicate the identical amino acid of all mentioned strains). 


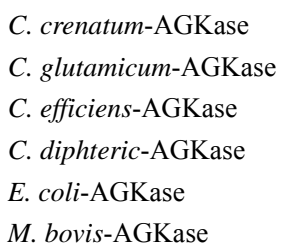

$\begin{array}{ll} & \\ & \\ \text { AVRGGVSAAHVIDGRIAHSVLLELLTMGG IGTMVL } & 293 \\ \text { AVRGGVSAAHVIDGRIAHSVLLELLTMGG IGTMVL } & 293 \\ \text { AVRGGVNAAHVIDGRIAHSVLLELLTMGG IGTMVL } & 293 \\ \text { AVI HGVSAAHV IDGRVAHSVLLELLTSGGVGTMVV } & 293 \\ \text { VNADQAATALAATLGADLILLSDVSGILDGKGQRIA } & 194 \\ \text { LRAVIGGVPSAHIIDGRVTHCVLVELFTDAGTGTKVV } & 292\end{array}$

Figure 7. AGKase shows feature of putative ATP-binding domain protein. (\# indicates a putative ATP-binding site).

\#\#

\begin{tabular}{|c|c|c|c|c|c|}
\hline E. coli-ArgR & \multicolumn{5}{|c|}{ KNLVLDIDYNDAVVVIHTSFGAAQLITARLLDS L GKAEGI LGTI AGDDT } \\
\hline C. crenatum-ArgR & DELLVSTDHSGNI AMLRTPPGAAQYI & ASFI DI & VGL & KE- & GTIAGDDT \\
\hline Consensus & $\begin{array}{llll}\mathrm{L} & \mathrm{D} & \mathrm{T} & \mathrm{GAAQ}\end{array}$ & A $\quad$ D & G & E & GTIAGDDT \\
\hline E. coli-ArgR & \multicolumn{2}{|l|}{ I FTTPANG FTVKALYEAILELFDQEL } & \multicolumn{3}{|c|}{156} \\
\hline C. crenatum-ArgR & \multicolumn{2}{|l|}{ VFVLARDPLTGKELGELLSG- -RTT } & \multicolumn{3}{|c|}{171} \\
\hline Consensus & K L E & & & & \\
\hline
\end{tabular}

Figure 8. Comparison of amino acids sequence of C-terminal domain of ArgR between C. crenatum and E. coli (\# indicates asparagine).

region binding arginine is quite consistent. The result implied that the two genes originated by a duplication of some common ancestral gene. Although it was modified and changed by different host in far-flung evolvement course, the partial region determining function still kept highly conservative.

\section{ACKNOWLEDGEMENTS}

The authors are thankful to the financial support from the National Natural Science Foundation of China (No. 30960012).

\section{REFERENCES}

[1] Gigot, D., Caplier, I., Str Vehary, S., Pavel, P., Michele, L. and Osberg, D. (1987) Amino-proximal sequences of the $\arg F$ and $\operatorname{argI}$ ornithine carbamoyltransferases from Escherichia coli K-12. Archives Internationales de Physiologie de Biochimie et de Biophysique, 86, 913-915.

[2] Sakanyan, V., Petrosyan, P., Lecocq, M., Boyen, A., Legrain, C., Demarez, M., Hallet, J.N. and Glansdorff, N. (1996) Genes and enzymes of the acetyl cycle of arginine biosynthesis in Corynebacterium glutamicum: Enzyme evolution in the early steps of the arginine pathway. Microbiology, 142, 99-108.

doi:10.1099/13500872-142-1-99

[3] Unin, R. and Glansdorff, N. (1986) Biosynthesis and metabolism of arginine in bacteria. Microbiology Reviews, 50, 314-352.

[4] Haas, D. and Kurer, V. (1982) N-acetylglutamate synthetase of Pseudomonas aeruginosa. An assay in vitro and feedback inhibition by arginine. European Journal of Biochemistry, 31, 290-295. doi:10.1111/j.1432-1033.1972.tb02531.x

[5] Heimberg, H., Boyen, A., Crabeel, M. and Glansdorff, N. (1990) Escherichia coli and Saccharomyces cerevisiae acetylornithine aminotransferase: Evolutionary relationship with ornithine aminotransferase. Gene, 90, 69-78.

[6] Hindle, Z. and Callis, R. (1994) Cloning and expression in Enterobacteria coli of a Streptomyces coelicolor A3(2) $\operatorname{argCJB}$ gene cluster. Microbiology, 140, 311-320. doi:10.1099/13500872-140-2-311

[7] Shengdong, L. (1999) Experiment technology of molecule biology. China Consonancy Medical University Publishing Company, Beijing.

[8] Maas, W.K. (1994) The arginine repressor of Escherichia coli. Microbiology Reviews, 58, 631-640.

[9] Miller, C.M., Baumberg, S. and Stockley, P. G. (1997) Operator interactions by the Bacillus subtilis arginine repressor/activator, AhrC: Novel positioning and DNAmediated assembly of a transcriptional activator at catabolic sites. Molecular Microbiology, 26, 37-48. doi:10.1046/j.1365-2958.1997.5441907.x

[10] Rodriguez-Garcia, A., Ludovice, M., Martin, J.F. and Liras, P. (1997) Arginine boxes and the $\operatorname{argR}$ gene in Streptomyces clavuligerus: Evidence for a clear regulation of the arginine pathway. Molecular Microbiology, 25, 219-228. doi:10.1046/j.1365-2958.1997.4511815.x

[11] Maghnouj, A. and Sousa, C.T.F. (1998) The $\operatorname{arcABDC}$ gene cluster, encoding the arginine deiminase pathway of Bacillus licheniformis, and its activation by the arginine repressor argR. Journal of Bacteriology, 180, 6468-6475.

[12] Dion, M. and Chalier, D. (1997) The highly thermostable arginine repressor of Bacillus stearothermophilus: gene cloning and repressor-operator interactions. Molecular Microbiology, 25, 385-398. doi:10.1046/j.1365-2958.1997.4781845.x

[13] Kira, S.M., Andrey, A.M. and Mikhail S.G. (2001) Conservation of the binding site for the arginine repressor in all bacterial lineages. Genome Biology, 2, 0013.1-0013.8.

[14] Cerdeño-Tárraga, A.M., Efstratiou, A., Dover, L.G., Holden, M.T., Pallen, M., Bentley, S.D., Besra, G.S., 
Churcher, C., James, K.D., De Zoysa, A., Chillingworth, T., Cronin, A., Dowd, L., Feltwell, T., Hamlin, N., Holroyd, S., Jagels, K., Moule, S., Quail, M.A., Rabbinowitsch, E., Rutherford, K.M., Thomson, N.R., Unwin, L., Whitehead, S., Barrell, B.G. and Parkhill, J. (2003) The complete genome sequence and analysis of Corynebacterium diphtheriae NCTC13129. Nucleic Acids Research, 22, 6516-6523. doi:10.1093/nar/gkg874

[15] Nishio, Y., Nakamura, Y., Kawarabayasi, Y., Usuda, Y., Kimura, E., Sugimoto, S., Matsui, K., Yamagishi, A., Kikuchi, H., Ikeo, K. and Gojobori, T. (2003) Comparative complete genome sequence analysis of the amino acid replacements responsible for the thermostability of Corynebacterium efficiens. Genome Research, 13, 15721579. doi:10.1101/gr.1285603

[16] Cole, S.T., Brosch, R., Parkhill J., Garnier, T., Churcher, C., Harris, D., Gordon, S.V., Eiglmeier, K. and Gas, T. F. S. (1998) Deciphering the biology of Mycobacterium tuberculosis from the complete genome sequence. Nature, 393, 537-544. doi:10.1038/31159

[17] Schell, M.A., Karmirantzou, M., Snel, B., Vilanova, D. Berger, B., Pessi, G., Zwahlen, M.C., Desiere, F., Bork, P., Delley, M., Pridmore, R.D. and Arigoni, F. (2002) The genome sequence of Bifidobacterium longum reflects its adaptation to the human gastrointestinal tract. Proceedings of the National Academy of Sciences of the United States of America, 99, 14422-14427. doi:10.1073/pnas.212527599

[18] Parkhill, J., Achtman, M., Bentley, S.D., Churcher, C., Klee, S.R., Morelli, G., Basham, D., Brown, D., Chillingworth, T., Davies, R.M., Davis, P., Devlin, K., Feltwell, T., Hamlin, N., Holroyd, S., Jagels, K., Leather, S., Moule, S., Mungall, K., Quail, M.A., Rajandream, M.A., Rutherford, K.M., Simmonds, M., Skelton, J., Whitehead, S., Spratt, B.G. and Barrell, B.G. (2000) Complete DNA sequence of a serogroup A strain of Neisseria meningitidis Z2491. Nature, 404, 502-506. doi:10.1038/35006655

[19] Ghochikyan, A., Karaivanova, I.M., Lecocq, M., Vusio, P., Arnaud, M.C., Snapyan, M., Weigel, P., Guével, L., Buckle, M. and Sakanyan, V. (2002) Arginine Operator Binding by Heterologous and Chimeric ArgR Repressors from Escherichia coli and Bacillus stearothermophilus. $J$ Bacteriol, 184, 6602-6614. doi:10.1128/JB.184.23.6602-6614.2002 